\title{
DIAGNÓSTICO DE LA SITUACIÓN DE LAS VISITAS A PACIENTES RECLUIDOS EN UNIDADES DE CUIDADOS INTENSIVOS
}

\section{DIAGNOSIS OF THE SITUATION OF VISITS TO PATIENTS HELD IN INTENSIVE CARE UNITS}

\section{TITULO CORTO: DIAGNÓSTICO DE LA SITUACIÓN DE LAS VISITAS A PACIENTES}

\author{
Rosa Del Socorro Morales-Aguilar ${ }^{1}$, Gloria Elena Lastre-Amell ${ }^{2}$, Alba Pardo-Vasquez ${ }^{3}$, \\ Moisés De La Hoz-Mercado ${ }^{4}$
}

Recibido en noviembre 09 de 2015

Aceptado en marzo 14 de 2016

\section{RESUMEN}

La visita es el espacio donde es posible que se relacionen el paciente, la familia y el personal de salud, cuando los pacientes están recluidos en las Unidades de Cuidados Intensivos. El propósito del artículo es diagnosticar la situación de las visitas a pacientes recluidos en unidades de cuidados intensivos de Instituciones Prestadoras de Salud (IPS) de Barranquilla. Es un estudio descriptivo, transversal; la población fueron cinco unidades de cuidados intensivos, la muestra intrínseca correspondió a 34 participantes. Los resultados identificados en los encuestados coincidieron en el horario de visitas de mañana y tarde $53 \%$; el tiempo de permanencia de los familiares durante la visita fue de media hora $30 \%$; el número de personas permitidos es de tres familiares $70 \%$; la información sobre la evolución del paciente la suministra el médico especialista $65 \%$; se evidenció debilidad del $18 \%$ en la solicitud del consentimiento informado al realizar los procedimientos. Estos resultados sirven para generar cambios en el futuro con la atención prestada a los familiares de pacientes críticos, basados en las recomendaciones de la Asociación Americana de Cuidados Intensivos en visitas más flexibles centradas en la relación paciente familia, con el fin de minimizar la ansiedad producida por la gravedad de su situación y el entorno de la Unidad de Cuidados Intensivos.

Palabras Clave: Enfermería; Cuidados Intensivos; Familia

\section{ABSTRACT}

The visit is the space where it is possible to relate the patient, family and health personnel. To diagnose the situation of the visits to patients imprisoned in units of intensive care of Institutions Prestadoras of Health (IPS). Study descriptive, cross; the population was five intensive care units, the intrinsic sample consisted of 34 participants. respondents agreed visiting hours morning and afternoon $53 \%$; the residence time of family members during the

\footnotetext{
1 Enfermera, Magister en Enfermería. Universidad Metropolitana. Barranquilla, Colombia. Correo: rosa.morales@unimetro.edu.co

2. Enfermera, Magister en Salud pública. Universidad Metropolitana. Barranquilla, Colombia. Correo: glastreamell@yahoo.es

3. Enfermera, Magister en Educación. Universidad Metropolitana. Barranquilla, Colombia. Correo: albacpardo@yahoo.es

4. Enfermero, Magister en Educación. Universidad Metropolitana. Barranquilla, Colombia. Correo: moisesjesus_@hotmail.com
} 
half-hour visit was $30 \%$; the number of people allowed is three relatives $70 \%$; information about the patient's progress is provided by the medical specialist $65 \% ; 18 \%$ weakness was evident in the application of informed consent procedures make. These results serve to generate changes in the future with the attention paid to the families of critically ill patients, based on the recommendations of the American Association of Intensive Care more flexible patient visits focused on family relationship, in order to minimize anxiety produced by the gravity of their situation and environment of the Unit of Intensive care.

Keywords: Nursing; Intensive Care; Family

\section{INTRODUCCIÓN}

L as Unidades de Cuidados Intensivos (UCI), son urecintos restringidos, donde el personal de salud mantiene poco contacto con familiares y allegados a los pacientes. Debido a esta situación, la relación en estos lugares se limita a los horarios de visita y al momento de la información médica; la regulación del ingreso a este servicio está dada por los profesionales de la salud, en particular las enfermeras ${ }^{1}$.

Es por ello que los pacientes ingresados a la UCI se encuentran enfrentados a factores estresantes, uno de ellos es la separación o aislamiento; durante la hospitalización del paciente, existen condiciones que pueden limitar esa situación, como son las restricciones generadas en algunas normas institucionales, condiciones de salud o de comportamientos del paciente ${ }^{2}$; asimismo, la estancia en estos servicios trae consigo una experiencia de vida de ansiedad, estrés, miedo, inseguridad y aislamiento tanto para la familia como para el paciente. Cabe considerar que un estudio reveló 45 necesidades concretas de los familiares que tienen pacientes en estado crítico, cuando estos están enfrentando un proceso de enfermedad, como brindar una información y atención de calidad, bienestar, horarios flexibles de visita, aspectos de entorno, entre otros; considerando así al profesional de enfermería, como el idóneo para cubrir mejor la mayoría de estas necesidades ${ }^{3}$.

Lo anterior indica que el diagnóstico de la situación de las visitas a pacientes recluidos en Unidades de Cuidados Intensivos es una necesidad importante, debido a que disminuyen todos estos factores, contribuyendo a mejorar la relación terapéutica entre la familia, paciente y personal de salud, para así favorecer la recuperación de la persona enferma ${ }^{4}$. Denotando un aspecto significativo, el papel de la familia ante estas situaciones de individuo o paciente en estado crítico, debido a que ayudan de manera significativa a la mejoría del paciente e incluso se les hace participe en la toma de decisiones con el equipo de salud, ya que son el punto de partida importante de apoyo y vínculo entre estos ${ }^{5}$.

Es más, la experiencia de padecer una enfermedad crítica y la hospitalización en la UCI de un paciente es dura, debido al sufrimiento que ocasiona por los efectos de la enfermedad, los procedimientos realizados y los elementos utilizados en el tratamiento para resolver la enfermedad; además, en los hospitales, donde la permanencia es condicionada por limitaciones en el número de personas, familiares y donde los horarios para el ingreso para visitar a los pacientes se restringen, la sensación de abandono es más profunda y el sufrimiento es mayor. Es por esto que los pacientes manifiestan sentirse solos, para muchos estar en la unidad de cuidados intensivos significa ser abandonado ${ }^{6}$.

Otro aspecto a resaltar es la importancia y la socialización de los protocolos en las Unidades de Cuidados Intensivos a los familiares, cuya finalidad es disminuir los temores por las situaciones vividas en estas, proporcionando así un ambiente de confianza ${ }^{4}$.

Con relación a la frecuencia de la información sobre la evolución del paciente, el profesional de salud en las UCI señala la importancia de la información para apoyar a la familia, mostrando gran disponibilidad informativa, comprensión y paciencia, donde es posible construir una vinculación saludable entre el paciente y la familia, aun en la situación crítica que están viviendo. Seguidamente, el autor refiere que la restricción de las visitas genera una situación de aislamiento, separación e incertidumbre entre el paciente y la familia? 
Considerando que el acto de cuidado se da a partir de la información o comunicación y relación interpersonal humanizada entre el profesional de enfermería y el sujeto de cuidado ${ }^{8}$, el rol de enfermería está llamado a liderar la situación de las visitas a pacientes de los familiares en las Unidades de Cuidados Intensivos. En este sentido la(el) enfermera(o) debe priorizar la humanización a la hora de organizar las visitas, con el fin de fortalecer la relación con los familiares de los pacientes críticos, para ello corresponde crear estrategias de flexibilización de las visitas tanto como personalizarlas de acuerdo a las necesidades de los familiares de los pacientes ${ }^{9}$. Los pacientes hospitalizados en la Unidad de Cuidados Intensivos, por padecer alguna patología que pone en riesgo su vida, necesitan, además, de los cuidados continuos e inmediatos, aislarse de la familia y enfrentarse a una situación desconocida ${ }^{7}$, esta situación aumenta el sufrimiento.

Este estudio es pertinente debido a la revisión y búsqueda bibliográfica realizada por los investigadores, no se encontró a nivel nacional y local estudios donde se aprecie sobre el tema, considerando así que los familiares buscan al personal de salud para que les comunique la evolución de su paciente y solicitan constantemente la entrada a la unidad para compartir momentos con el familiar enfermo ${ }^{2}$. Por lo anterior, el objetivo fue diagnosticar la situación de las visitas a pacientes recluidos en unidades de cuidados intensivos de Instituciones Prestadoras de Salud (IPS) de Barranquilla en un periodo de 4 meses.

\section{MATERIALES Y MÉTODOS}

El estudio fue descriptivo, de corte transversal, realizado desde febrero a mayo del año 2013. La población y muestra estuvo conformada por 34 personas: 8 médicos, 11 enfermeras y 15 auxiliares de enfermería de cinco unidades de cuidados intensivos de varias IPS de la ciudad de Barranquilla, de 10 UCI que se les invito a participar en el estudio, el tipo de muestreo fue aleatorio simple.

\section{Criterios de inclusión}

Médicos, enfermeras y auxiliares de enfermería que participan en la situación de las visitas a pacientes de familiares recluidos en UCI de IPS de Barranquilla y aquellos que por voluntad propia aceptaron participar en el estudio.

\section{Instrumentos}

Previo a la aplicación de los instrumentos se realizó una prueba piloto con 10 profesionales expertos en el área de contenido y metodológico, permitiendo mejorar su diseño original. Se aplicó un instrumento elaborado por los autores, con el objetivo de medir las variables sociodemográficas, diagnóstico de la situación de las visitas a pacientes recluidos en UCI, según horarios, tiempo de permanencia de los familiares en las visitas, número de familiares permitidos, frecuencia, información a los familiares sobre evolución, procedimiento y consentimiento informado. El instrumento estuvo conformado por un total de 23 ítems, los cuales eran preguntas de selección múltiple. Los datos obtenidos se tabularon y se analizaron con el programa estadístico SPSS versión 18, utilizando estadística descriptiva: porcentajes y frecuencia.

\section{Declaración de aspectos éticos}

Contó con la aprobación de la Coordinación de Investigación del Programa de Enfermería de la Universidad Metropolitana y con el permiso de las IPS para obtener la información pertinente. Además, la investigación tuvo en cuenta la Resolución N ${ }^{\circ} 008430$ de 1993, la Ley 911 de $2004^{10}$. La participación de las personas que respondieron a la recolección de la información fue voluntaria, mediante la firma del consentimiento informado. Para obtener la información sobre diagnóstico de la situación de las visitas a pacientes recluidos en UCI, se contactó a la presidente de la Asociación Regional de Cuidados Intensivos para identificar el número de IPS con unidad de cuidado intensivo, posteriormente con una carta dirigida a todos los responsables de las diferentes unidades, se solicitó el permiso para recolectar la información. Del total de las IPS invitadas para realizar la investigación, 5 respondieron autorizando el estudio.

\section{RESULTADOS}

El $76 \%$ de los profesionales de salud eran mujeres, con respecto al cargo en la unidad de cuidado intensivo se identificó que el $44 \%$ fueron auxiliares de enfermería, $32 \%$ enfermeras(os) y el $24 \%$ médicos.

Con relación al diagnóstico de la situación de las visitas a pacientes recluidos en UCI, se demostró que el 94\% de los profesionales respondieron que los familiares sí 
conocen el protocolo de visitas para el ingreso a la unidad de cuidados intensivos, lo que favorece la participación y compresión de la familia en la atención del enfermo.

En cuanto al horario de las visitas en las diferentes jornadas, se encontró que el $53 \%$ tiene visita tanto en la jornada de la mañana como en la tarde; según el tiempo de permanencia de los familiares con el paciente, se halló que el mayor porcentaje (40\%), está representado en 30 minutos de duración y el número de familiares permitidos por visitas, es de tres personas con el $70 \%$ (Tabla 1).

Tabla 1. Diagnóstico de la situación de las visitas a pacientes recluidos en unidades de cuidados intensivos, según Horario, tiempo, numero de familiares y frecuencia

\begin{tabular}{|c|c|c|}
\hline Variables & Frecuencia & Porcentaje \\
\hline Mañana & 9 & 26 \\
\hline Tarde & 5 & 15 \\
\hline Noche & 2 & 6 \\
\hline Mañana y Tarde & 18 & 53 \\
\hline Total & 34 & 100 \\
\hline \multicolumn{3}{|c|}{$\begin{array}{l}\text { Tiempo de permanencia de los familiares durante las visitas a sus pacientes hospitalizados en las unidades de } \\
\text { cuidados intensivos. }\end{array}$} \\
\hline Variables & Frecuencia & Porcentaje \\
\hline 15 minutos & 9 & 26 \\
\hline 30 minutos & 15 & 44 \\
\hline 45 minutos & 1 & 3 \\
\hline 60 minutos & 9 & 26 \\
\hline Total & 34 & 100 \\
\hline \multicolumn{3}{|c|}{ Numero de familiares permitidos en las visitas } \\
\hline Variables & Frecuencia & Porcentaje \\
\hline Uno & 6 & 18 \\
\hline Dos & 4 & 12 \\
\hline Tres & 24 & 70 \\
\hline Total & 34 & 100 \\
\hline \multicolumn{3}{|c|}{$\begin{array}{l}\text { Frecuencia de las visitas permitidas al día a los familiares de los pacientes hospitalizados en las unidades de } \\
\text { cuidados intensivos. }\end{array}$} \\
\hline Variables & Frecuencia & Porcentaje \\
\hline Una visita al día & 8 & 24 \\
\hline Dos visita al día & 20 & 59 \\
\hline Tres visita al día & 3 & 9 \\
\hline Cuando lo solicite & 3 & 9 \\
\hline Total & 34 & 100 \\
\hline
\end{tabular}


Asimismo, en cuanto a la frecuencia de las visitas permitidas al día a los familiares de los pacientes recluidos en las UCI, los resultados indican que el $59 \%$ permiten dos visitas al día. Infiriendo que la flexibilidad en la organización de las visitas es beneficiosa para el paciente y la familia, debido a que constituye en un momento de interacción del personal de salud con los familiares (Tabla 1).

Referente a la característica de la información brindada a los familiares, sé identifico que el $64 \%$ de la información sobre la evolución del paciente al familiar es suministrada por el médico especialista (Tabla 2).

En relación al medio por el cual se comunica la evolución del paciente a la familia, se halló que el personal de salud refiere que el $12 \%$ recibe información telefónica, en cuanto a la información escrita un 15\% señala recibirla; respecto a quien informa sobre los procedimientos que se le van a realizar al paciente, el $47 \%$ expresa que es el médico general; un $37 \%$ la enfermera (Tabla 2).

Con relación a la solicitud del consentimiento informado para los procedimientos que se realizan al paciente, un $76 \%$ de los profesionales de la salud manifiestan que si se les solicita el consentimiento informado. Analizando estas características, sobresalen las necesidades de los familiares sobre una buena y oportuna información debido a que se mejoraría la comunicación, entre el personal de salud y los familiares (Tabla 2).

Tabla 2. Diagnóstico de la situación de las visitas a pacientes recluidos en unidades de cuidados intensivos según la información brindada a los familiares

\begin{tabular}{|c|c|c|}
\hline Variables & Frecuencia & Porcentaje \\
\hline Especialista & 22 & 65 \\
\hline Médico general o residente & 8 & 24 \\
\hline Médicos y enfermeras & 4 & 12 \\
\hline Total & 34 & 100 \\
\hline
\end{tabular}

Frecuencia e información telefónica o escrita.

\begin{tabular}{|c|c|c|c|c|c|c|c|c|}
\hline \multicolumn{2}{|c|}{$\begin{array}{c}\text { FRECUENCIA DE LA } \\
\text { INFORMACIÓN }\end{array}$} & \multicolumn{3}{c|}{$\begin{array}{c}\text { SI LA INFORMACIÓN ES } \\
\text { TELEFÓNICA }\end{array}$} & \multicolumn{3}{c|}{ SI LA INFORMACIÓN ESCRITA } \\
\hline CRITERIOS & FR. & $\%$ & CRITERIOS & FR. & $\%$ & CRITERIOS & FR. & $\%$ \\
\hline Una vez & 15 & 44 & Sí & 0 & 0 & Si & 5 & 15 \\
\hline Dos veces & 15 & 44 & No & 30 & 88 & No & 29 & 85 \\
\hline Lo soliciten & 4 & 12 & excepciones & 4 & 12 & & & \\
\hline Total & 34 & 100 & Total & 34 & 100 & Total & 34 & 100 \\
\hline
\end{tabular}

Información del equipo de salud sobre los procedimientos a realizar a los pacientes.

\begin{tabular}{|c|c|c|}
\hline Variables & Frecuencia & Porcentaje \\
\hline Especialista & 6 & 18 \\
\hline Médico general & 16 & 47 \\
\hline Enfermera & 12 & 35 \\
\hline Auxiliar de enfermería & 0 & 0 \\
\hline Total & 34 & 100 \\
\hline
\end{tabular}

Solicitud del Consentimiento informado antes de los procedimientos.

\begin{tabular}{|c|c|c|}
\hline Variables & Frecuencia & Porcentaje \\
\hline $\mathrm{Si}$ & 26 & 76 \\
\hline No & 6 & 18 \\
\hline Algunas veces & 2 & 6 \\
\hline Total & 34 & 100 \\
\hline
\end{tabular}




\section{DISCUSIÓN}

En la investigación, el personal de la salud en las Unidades de Cuidados Intensivos de Barranquilla que participaron activamente en la visita familiar fueron enfermeras, auxiliares de enfermería y médicos, observándose que el mayor porcentaje del personal de salud estuvo representado por las auxiliares de enfermería; en cuanto al género, el que mayor prevaleció fue el femenino. En el estudio de Santana et $\mathrm{al}^{11}$, identificaron 69 profesionales sanitarios de la UCI 58 eran enfermeras(os) y 11 médicos; datos similares en el de médicos, pero no en el equipo de enfermeras. Al comparar los resultados de esta investigación, con relación al personal de salud que participa en las visitas, es similar al trabajo de Concha et $a l .{ }^{1}$, que refiere, las unidades de cuidados intensivos de adultos en España, están regulados por los profesionales, en particular las enfermeras.

Respecto al diagnóstico de la situación de las visitas a pacientes recluidos en unidades de cuidados intensivos, según horario, tiempo, numero de familiares y frecuencia, los profesionales de salud no suministra esta información a familiares de los pacientes, ósea no hay un manual o protocolo que se le entregue a los a los familiares de pacientes recluidos en UCI; difieren estos resultados, con el estudio de Abizanda et al ${ }^{12}$ debido a que ellos si entregan un manual informativo en donde se encuentra la información de la unidad de cuidados intensivos como la descripción del espacio físico del cubículo, horarios de visitas, nombre del médico, jefe del servicio, números telefónicos del servicio y del profesional de salud, horarios de información del estado de salud del paciente, este manual informativo se lo entregan a los familiares de los pacientes cuando este es ingresado a la unidad o sino cuando le solicita la información.

De igual forma Riani Llano ${ }^{13}$ relata que el entorno de la UCI, es fácil caer en errores de atención despersonalizada y se prevén aspectos sencillos como la buena comunicación, este ambiente puede parecer más amable, si la familia recibe una inducción, momento en que se debe explicar la modalidad de trabajo, facilitar información específica, debido a que es de gran ayuda. Y según Frizon et a ${ }^{14}$ donde refiere que el estar hospitalizado un individuo en una UCI, considera que es un momento difícil para la familia, ya que ellos enfrentan diferentes situaciones, sentimientos, proyectos de vida que implican a los familiares.

Referente a los horarios y visitas de los familiares a los pacientes en las unidades de cuidados intensivos, está información es establecida y brindada una sola vez al día a los familiares según Hidalgo et $\mathrm{a}^{15}$; comparando el presente estudio, se evidenció diferencia en relación con el horario, debido a que el personal de salud, indicó que el horario de visitas es mañana y tarde, o sea que tienen establecidos dos visitas al día; estos resultados son favorables para la familia porque así están visitando, observando y conociendo cómo va evolucionando su paciente y este criterio es básico para ellos, para saber si su paciente ha avanzado positiva o negativamente.

En cuanto al diagnóstico de la situación de las visitas a pacientes recluidos en UCI, según el tiempo de permanencia de los familiares, en esta investigación se determinó que el mayor porcentaje, eligieron el criterio de duración de visitas es de media hora, dato que coincide con el estudio, en donde se mostró que la duración de las visitas en las unidades de cuidados intensivos solía ser de media hora en la mayoría de los casos y el $12 \%$ a 1 hora ${ }^{16}$.

Asimismo, investigaciones descriptivas sobre las normas de las visita identificaron que solo se admite un visitante por paciente en la unidad del pacientes, el cual permite el contacto directo con él, debido a que los otros familiares solo pueden acceder al pasillo y observar a su familiar a través de un cristal, resultando que el 56\% de los encuestados dijeron que un solo familiar dentro de la habitación durante la visita era suficiente, el $41 \%$ de pacientes y el $37 \%$ de familiares lo consideraron insuficiente. El 60\% de la muestra conceptuó que dos familiares fuera de la habitación eran aptos; el $61 \%$ de enfermeras dudó, mientras que el $45 \%$ de médicos lo consideró insuficiente ${ }^{17}$. Al confrontar el estudio no se encontró coincidencia en los resultados, debido a que el personal de salud opino que tienen establecido que son tres familiares por paciente los que pueden entrar a visitar y que esto es suficiente, aclarando que no todos entran juntos a visitar sino uno por uno y todos los familiares permitidos en el ingreso tienen contacto con el paciente.

Con respecto al funcionario del equipo de salud que informa a los familiares sobre la evolución del paciente en el diagnóstico de la situación de las visitas, se evidenció en mayor porcentaje que el profesional de salud quien comunica es el especialista, seguido de los médicos generales y enfermeras(os) juntos, comparando estos resultados con el estudio de Arricivita et $\mathrm{a}^{18}$ en el cual se identifica similitud en donde la investigación mostró que el médico (86,36\%) es exclusivamente quien comunica a los familiares sobre la evolución del paciente y sólo en tres centros ( 2 en Tenerife y 1 en Gran Canaria) encontraron que el profesional de enfermería afirma participar conjuntamente con el médico en este proceso. 
Es más, el rol del profesional de enfermería es importante para el paciente y la familia, debido a que el profesional brinda atención en salud, cuidado directo, apoyo emocional al paciente, consejería a los familiares, especialmente en las unidades de cuidados intensivos ${ }^{19}$. Teniendo en cuenta que el entorno de la UCI plantea muchos desafíos, identificando semejanza con el estudio de Concha et $\mathrm{al}^{1}$, donde describió que el punto de partida para realizar la investigación sobre la relación entre enfermeras de UCI y familiares observaron que las enfermeras en el momento de la visita de familiares, el contacto es mínimo entre ellos, también las enfermeras se dedican a realizar otras ocupaciones sin tenerlos en cuenta a ellos. Igualmente, en otro estudio se identificó la importancia de que la enfermera informara al paciente y familiares sobre el estado de salud ${ }^{20}$. Es por eso que, en el momento del diagnóstico de la situación de las visitas a pacientes recluidos en UCI, es significativo realizar una interacción entre el personal de salud, familiares y pacientes, especialmente enfermería ${ }^{1}$.

De los resultados del tipo de información que se brinda en forma escrita o telefónica, el mayor porcentaje de los encuestados expresaron que la información se brinda vía telefónica y en menos proporción de forma escrita. Los datos anteriores afianzan lo referido por Velasco et $\mathrm{al}^{16}$ donde determinaron que en las unidades utilizan medio escrito como apoyo a la información y respecto a la información telefónica se restringe brindarla al familiar.

Por otra parte, en la investigación el médico es quien informa al familiar acerca de los procedimientos que se le realizan al paciente, seguido de las enfermeras, resultado semejante al de Concha et $\mathrm{al}^{1}$, quien en su artículo reporta que la información sobre evolución, procedimiento y estado de salud del paciente siempre esta competencia ha sido del médico; con respecto a las enfermeras, en el estudio de Pérez et al ${ }^{21}$ se express que la correlación y la información con el profesional de enfermería evaluaron bien este aspecto, en donde agradecían y los comprendían en situaciones difíciles. Analizando que el rol de la enfermera es el cuidado directo del paciente o perfil asistencial, administrativo/gerencial y educativo.

22 Con base en la variable de consentimiento informado, se demostró que el $76 \%$ de los encuestados, eligieron que sí solicitan el consentimiento informado a los pacientes y familiares sobre los procedimientos a realizarle al paciente en la UCI. En la revisión bibliográfica no se encontraron estudios que contrasten estos resultados, sin embargo, en el artículo de Solsona y Saralegui ${ }^{19}$. Se afirma "que el consentimiento informado se otorgará por escrito en los casos siguientes: intervención quirúrgica, procedimientos diagnósticos y terapéuticos invasores y, en general, cuando se vaya a realizar procedimientos que supongan riesgos o inconvenientes de notoria y previsible repercusión negativa en la salud del paciente".

Por lo anterior, se destaca en cuanto a diagnosticar la situación de las visitas a pacientes recluidos en unidades de cuidados intensivos de Instituciones Prestadoras de Salud (IPS) de Barranquilla como aspectos positivos de la investigación, lo siguiente: existencia de protocolo de visitas, estandarización con estudios sobre horario, número de visitas, tiempo y familiares permitidos a la visita, adquiriendo gran rigor en su similitud con estudios referentes de este estudio. Con relación a los aspectos negativos, se destaca la información sobre el consentimiento informado para la solicitud de los procedimientos, en el cual se debe obtener el cien por ciento de autorización, con respecto a la información suministrada a los familiares la cual debe ser suministrada siempre que el familiar lo solicite. Cabe resaltar que estos aspectos no se pudieron contrastar en el medio local, por no encontrarse información sobre el tema.

\section{CONCLUSIÓN}

Esta investigación evidenció que en las condiciones de las visitas de los familiares en las unidades de cuidados intensivos de las diferentes IPS de la ciudad de Barranquilla estudiadas la mayoría de los trabajadores de salud tenían conocimiento sobre el protocolo de ingreso para las visitas; otra condición identificada fue de 2 visitas permitidas al día, en la jornada de la mañana y tarde; en cuanto al tiempo de duración de la visita con el paciente fue de 30 minutos; con relación al número de familiares permitidos en a la visita al paciente se encontró 3 familiares por paciente.

Estos resultados sirven para generar cambios en el futuro con la atención prestada a los familiares de pacientes críticos, basados en las recomendaciones de la Asociación Americana de Cuidados Intensivos en visitas más flexibles centradas en la relación paciente-familia; además, los cuales deben basarse en la humanización, comunicación terapéutica para fomentar un ambiente de seguridad y confianza en la prestación del servicio, especialmente durante la visita familiar, con el fin de minimizar la ansiedad producida por la gravedad de su situación y el entorno de la UCI, en el paciente y la familia, mejorando la comunicación entre la familia y el personal de la salud, 
incrementar la satisfacción del paciente, familia y equipo de salud, lo cual redunda en la calidad del servicio y la contribución en la recuperación del paciente.

Durante el estudio se encontraron limitaciones de tiempo de los asistenciales para proveer información y el reglamento de las unidades de cuidados intensivos para acceder a ellas y la sugerencia que resulta de la investigación, es la relacionada con los aspectos que requieren los familiares y pacientes en el momento de la visita familiar para disponer de mejores experiencias en las unidades de cuidado crítico.

\section{AGRADECIMIENTOS}

A la Médico Internista, Intensivista, Epidemióloga Nelly Beltrán de la Universidad del Norte. Presidente de la Asociación colombiana de cuidado crítico y cuidado intensivo regional Costa Atlántica.

A las estudiantes de VIII semestre: Niebles Vallejo L.M.., Molina Villanueva S., Peláez Reinoso S.S., Plaza Rodríguez X. de la Universidad privada de Barranquilla, que participaron como auxiliares en la investigación.

\section{DECLARACION SOBRE CONFLICTOS DE INTERES}

Los autores declaran no tener ningún conflicto de intereses.

\section{REFERENCIAS BIBLIOGRÁFICAS}

1. Concha Zaforteza D, Sánchez Cuenca P, De Pedro J, Lastra P. Relación entre enfermeras de unidades de cuidados intensivos y familiares: indicios para el Cambio. Nure Investigación. 2004 Mar; (3):1-7.

2. Vanegas BC, Vega SL, Barbosa MF, González GM, Montoya JJ. Experiencias de profesionales de enfermería en el cuidado psicoemocional a pacientes en la unidad de cuidados intensivos, 2006. Revista Colombiana de Enfermería. 2008; 3(3): 21-31.

3. Jiménez Ocampo V, Zapata Gutiérrez L, Díaz Suárez L. Capacidad de afrontamiento y adaptación de los familiares del paciente adulto hospitalizado en una unidad de cuidado intensivo. Aquichan. 2013; 3(2):159-172.

4. Basco Prado L, Fariñas Rodríguez S, Hidalgo Blanco MÁ. Características del sueño de los pacientes en una unidad de cuidados intensivos. Rev Cubana Enfermer. 2010 Jun; 26(2): 44-51.
5. Gómez Martínez S, Ballester Arnal R, Gil Juliá B. El Cuestionario de Necesidades de los Familiares de Pacientes de Cuidados Intensivos (CCFNI) versión breve: adaptación y validación en población española. An. Sist. Sanit. Navar. 2011 Sep- Dic; 34 (3): 349-361.

6. Beltran Salazar OA. La experiencia de estar hospitalizado en una unidad de cuidado intensivo. Aquichan. 2009; 9 (1): $23-37$.

7. Cabezudo Torres M, Cantuarias Noriega N. Vivencias de los familiares de pacientes con ventilador mecánico en la Unidad De Cuidados Intensivos. Estudio fenomenológico. In Crescendo. 2011Jun; 2 (1):79-95.

8. Colombia. Ministerio de Educación Nacional. Ley 911 de 2004 por la cual se discan disposiciones en materia de responsabilidad deontológica para el ejercicio de la profesión de Enfermería en Colombia; se establece el régimen disciplinario correspondiente y se dictan otras disposiciones. [Internet]. Bogotá: [Boletín Oficial del Estado], numero 45.693. Oct 6 2004. Diario Oficial, 45693 (Oct. 6 2004)

9. Gálvez González M, Fernández Luque C, Muñumel Alameda G, Ríos Gallego F, Fernández Vargas L, Hidalgo B. Acompañamiento familiar: una herramienta para dignificar el proceso de muerte en la unidad de cuidados intensivos. Index Enferm. 2011 Dic; 20(4): 233-237.

10. Colombia. Ministerio de Salud. Resolución N ${ }^{\circ} 008430$ de 1993 Por la cual se establecen las normas científicas, técnicas y administrativas para la investigación en salud. [Internet]. Bogotá. (4 de Octubre de 1993). Disponible en: https://www.unisabana.edu.co/ fileadmin/Documentos/Investigacion/comite_de_etica/ Res_8430_1993_-_Salud.pdf

11. Santana Cabrera L, Sánchez Palacios M, Hernández Medina E, García Martul M, Robaina E, Villanueva Ortiz Á. Necesidades de los familiares de pacientes de Cuidados Intensivos: percepción de los familiares y del profesional. Rev. Med Intensiva. 2007 Sep; 31 (6):273-80.

12. Abizanda Campos R, Bernat Adell A, Ballester Arnal R, Bisbal Andrés E, Vidal Tegedor B, Cubedo Bort $M$, et al. Estrategias de información en una Unidad de Cuidados Intensivos polivalente. Med. Intensiva [revista en la Internet]. 2008 [citado 2014 Mayo 30]; 32(5): 216-221. Disponible en: http://scielo. isciii.es $/$ scielo.php? script $=$ sci_arttext \&pid $=$ S0210$56912008000500002 \& \operatorname{lng}=$ es

13. Riani Llano N. Momentos clave para humanizar el paso por la unidad de cuidados intensivos neonatal. Revista Pers. Bioet. 2007 Dic; 11(29):138-145.

14. Frizon G, Nascimento ER, Bertoncello KC, Martins JJ. Familiares na sala de espera de uma unidade de terapia intensiva: sentimentos revelados. Rev Gaúcha Enferm. 2011 Mar; 32(1):72-8.

15. Hidalgo Fabrellas I, Vélez Pérez Y, Pueyo Ribas E. Qué es importante para los familiares de los pacientes de una Unidad de Cuidados Intensivos. Enferm Intensiva. 2007 Jul; 18(3):106-14. 
16. Velasco Bueno JM, Prieto de Paula JF, Castillo Morales J, Merino Nogales N, Perea-Milla LE. Organización de las visitas de familiares en las unidades de cuidados intensivos en España. Enferm Intensiva. 2005 Abr; 16(2):73-83.

17. Burgos Naranjo RM, Cano Peregrina MA, García Córdoba C, Lage Lopez A, Lopez Pelegri M. Visitas de familiares a pacientes ingresados en la Unidad de Cuidados Coronarios: Opinión de todos los implicados. Enfermería en Cardiología. 2000 Ago; 7(20):30-41.

18. Arricivita Verdasco AM, Cabrera Figueroa J, Arias Hernández MM, Robayna Delgado MC, Díaz Rodríguez L. Características de la organización de las visitas en las unidades de cuidados críticos de la comunidad autónoma de canarias. Enfermería en Cardiología 2002 Abr; 25: 38-42.
19. Solsona Durán JF, Saralegui Reta I. En los servicios de medicina intensiva se debe solicitar consentimiento informado para la transfusión no urgente. Med. Intensiva [revista en la Internet]. 2009 Abr [citado 2014 Mayo 24]; 33(3): 149-149. Disponible en: http://scielo. isciii.es $/$ scielo.php? script $=$ sci_arttext $\&$ pid $=$ S0210$56912009000300009 \& \operatorname{lng}=$ es

20. Navarro Arnedo J. Revisión de los estudios sobre los profesionales de enfermería de las Unidades de Cuidados Intensivos de España. Revista Global [revista en la Internet]. 2012 Abril [citado 2015 Noviembre 30]; 26: 267-289. Disponible en: www.um.es/global/

21. Pérez Cárdenas M, Rodríguez Gómez M, Fernández Herranz A, Catalán González M, Montejo González J. Valoración del grado de satisfacción de los familiares de pacientes ingresados en una unidad de cuidados intensivos. Med Intensiva. 2004; 28 (5):237-49.

Para citar este artículo: Morales-Aguilar R, Lastre-Amell GE, Pardo-Vásquez A, De La HozMercado M. Diagnóstico de la situación de las visitas a pacientes recluidos en unidades de cuidados intensivos. Duazary. 2017 enero; 14 (1): 16 - 24 